\title{
Soft pneumatic prosthetic hand
}

\author{
Jan Fras and Kaspar Althoefer *
}

October 17, 2018

\begin{abstract}
Conventional prosthetic devices are heavy, expensive and rigid. They are complex, fragile and require sophisticated control strategies in order to deal with the grasping and manipulation tasks. In this paper, we propose a new pneumatic soft prosthetic hand that is very simple to control due to its compliant structure and cheap in production. It is designed to be easily reshaped and resized to adapt easily to each individual user preferences. It is designed to be frequently changed whenever a child patient requires a bigger size or whenever the old one is worn out or broken. Since it is soft and compliant it can be safely used even by small children without a risk of harmful mechanical interaction.
\end{abstract}

\section{Introduction}

The human hand is a very complex structure that contains 27 degrees of freedom. It is strong but precise, compliant and dexterous. For that reason, the conventional prosthetic hands are complex and expensive devices. They contain sophisticated electrical motors and require complex controllers. They are heavy and incontinent. They are made of rigid components, so any compliance is ensured with an even more complex controller or with more advanced actuators. Taking all the drawbacks of traditional rigid prosthetics into account, soft robotics seems to be a promising approach to introduce a significant improvement into that area. Soft hands can be significantly cheaper as they are made of simple and affordable materials. Since soft devices are compliant they adapt easily to the environment and thus reduce the complexity of the controller. That reduces the costs further, as for many grasps even one degree of actuation is sufficient. For that reason, they can be considered more suitable for children users as they require frequent prosthetic change due to their body growth. In recent years a number of soft and compliant hand designs has been presented.

${ }^{\star}$ J. Fras and K. Althoefer are with the Centre for Advanced Robotics @ Queen Mary (ARQ), Faculty of Science and Engineering, Queen Mary University of London, j.fras@qmul.ac.uk, k.althoefer@qmul.ac.uk 
Pisa/IIT Soft Hand [1] uses only one motor to actuate all the joints which significantly simplifies the control. In [2] a soft hand composed of pneumatic PneuFlex actuators is proposed. In that particular design, each finger is made of an individually controlled pneumatic actuator. Another soft pneumatic hand is proposed in [3]. Its mechanics is quite similar to [2] but have sensors integrated. There are various designs utilizing a different number of actuators driven by tendons or pressure [4-6]. All of those designs propose a morphology that is similar to a human hand, however, they differ a lot form the actual human limb in terms of shape and appearance, which is a significant factor when the device is considered as a prosthetic [7]. In this paper, we propose a pneumatic prosthetic that has truly anatomical shape and is much more human-like than other pneumatic designs presented so far. The hand provides six degrees of actuation that can be controlled separately or connected together. The thumb in the proposed device is designed to work in both the apposition and opposition modes.

The hand dimensions, proportions, and configuration can be easily adjusted for each and every individual user. It is made of soft and compliant materials and due to that it is safe and adapts easily to the handled object. It is designed to be a low cost in order to make it affordable for a wide range of patients and in particular to make it easily changeable for children patients that require a frequent prosthetic change due to their body growth. It is designed to use simple materials and common techniques in order to allow a low qualified user to manufacture it to make it even more accessible. The manufacturing process utilizes $3 \mathrm{D}$ printing and silicone casting techniques.

\section{Design}

The hand is made out of soft materials. The palm and inner side of fingers are made of relatively stiff silicone SmoothSill 940 (Shore A 40). The actuators embedded in fingers are made of soft silicone EcoFlex 0050 (Shore 00 50) and a polyester thread. Combination of materials with different mechanical properties enables to achieve the required bending motion. The design is derived from soft industrial grippers presented in [8]. Previous research has shown that the proposed approach can be efficiently used in grasping and manipulation tasks, see fig. 1.

\subsection{Hand structure}

In order to make the prosthetic as bio-mimicking as possible, its design is based on a $3 \mathrm{D}$ scan of real human hand. Such an approach ensures the proper ratio of the fingers and the palm. The fingers are driven with soft fluidic actuators, one per finger and two for the thumb. The actuators are hollow chambers reinforced with polyester thread. The thread constrains the fingers radial expansion but does not affect their elongation capabilities. The stiff silicone creates an exoskeleton that defines the hand shape and affects the deformation of the actuators, fig. 2. Since the exoskeleton is made of stiff silicone it tends to elongate 

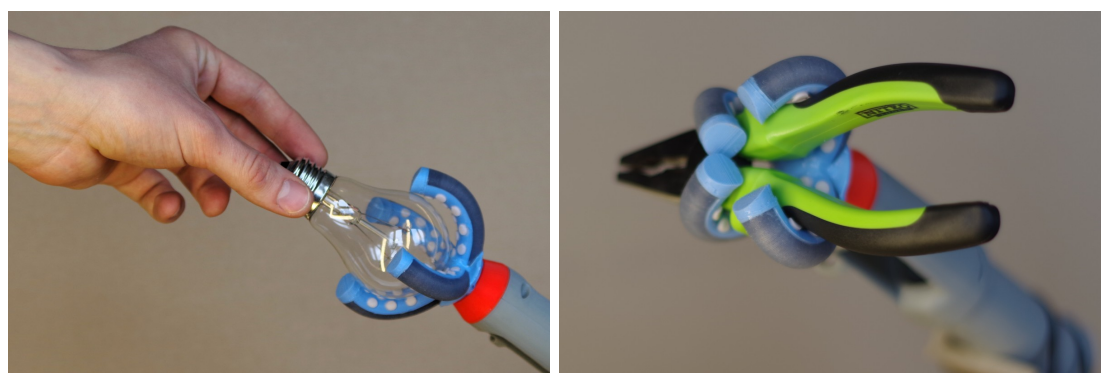

Fig. 1: Soft industrial gripper [8].

less than the actuator itself and thanks to that the translational motion of the actuators is transformed into the bending motion of the finger. The exoskeleton structure is designed to constrain the actuators deformation more in the areas that correspond with the bones and to emphasize the bending in the areas corresponding with the joints of the real human fingers.

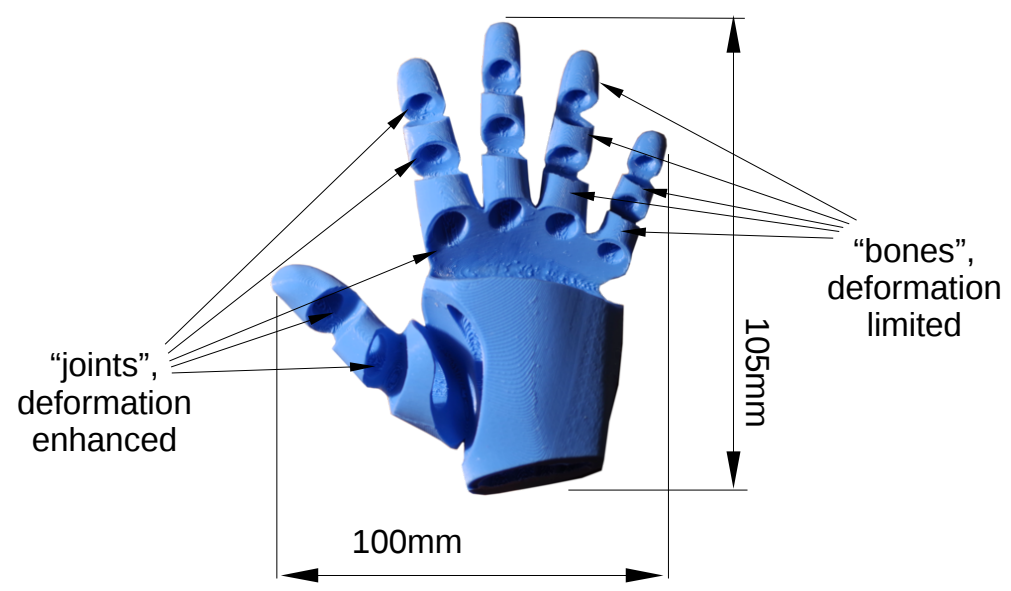

Fig. 2: Exoskeleton. Defines the hands' shape and generates bending motion.

One actuator is used for each finger except the thumb that is controlled with two actuators. Thanks to that its configuration can be changed between opposition and opposition mode.

Despite the fact exoskeleton constrains the motion of the actuators and reduces it in the link areas, the whole design is soft and compliant. For that reason, the fingers' motion is not limited to discrete joints but is distributed on along their lengths. In previous research, we have shown that finite rotary joints can be 


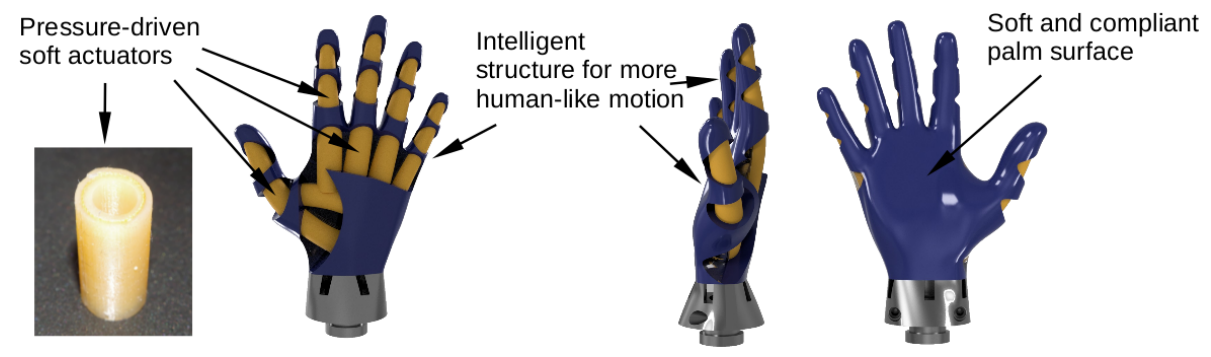

Fig. 3: Structure of the hand

made using only soft materials [9], but we found such a solution not applicable in that case due to excessive size and the need of using separate actuator for each joint. Using such actuators would make the design and the manufacturing unnecessarily too complex.

\subsection{Actuator}

The cross-section of the actuator is circular that ensures its consistency during the actuation process. Any other cross-section geometry would be affected by the internal pressure [9]. The actuators are pneumatic flexible cylinders that contain helical reinforcement into their walls [10]. They are driven by the pneumatic pressure that causes them to expand. Since the reinforcement constrains any radial deformation they expand only in longitudinal direction. Such elongation is then converted into bending motion by the exoskeleton structure [8]. We have already successfully embedded similar actuators for grasping, manipulation and locomotion [8-10]. Each actuator can be controlled independently but due to their flexibility and compliance, they can also efficiently work in groups. Such a property makes the dexterous manipulation less complex in terms of control and allows the hand structure to take over part of the controller's effort.

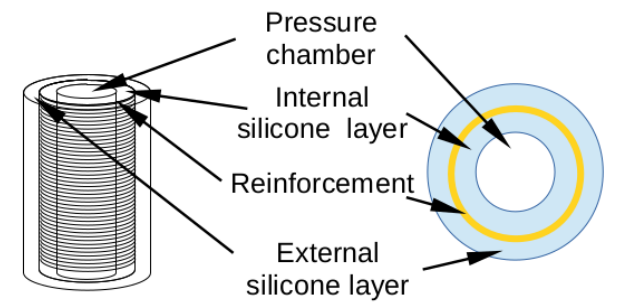

Fig. 4: Structure of the actuator

The actuator's structure is presented in fig. 4. It contains an internal wall that surrounds the actuation volume and creates the actuation chamber. The 
force generated by internal pressure is transferred by this wall on a surrounding reinforcement. The reinforcement is made with single polyester thread that forms a helix around the pressure chamber. Small thread pitch angle guarantees that the reinforcement does not affect the longitudinal actuator properties, but efficiently constrains radial deformation [10]. The external wall of the actuator enforces the reinforcement to stay in the desired shape and to not detach from the actuation chamber. The tip and the bottom of the actuator are sealed with a stiffer material (same as used for the exoskeleton structure). Bottom of the actuator contains small, $1.2 \mathrm{~mm}$ wide channel that is used to connect the pressure pipes.

\subsection{Mechanical interface}

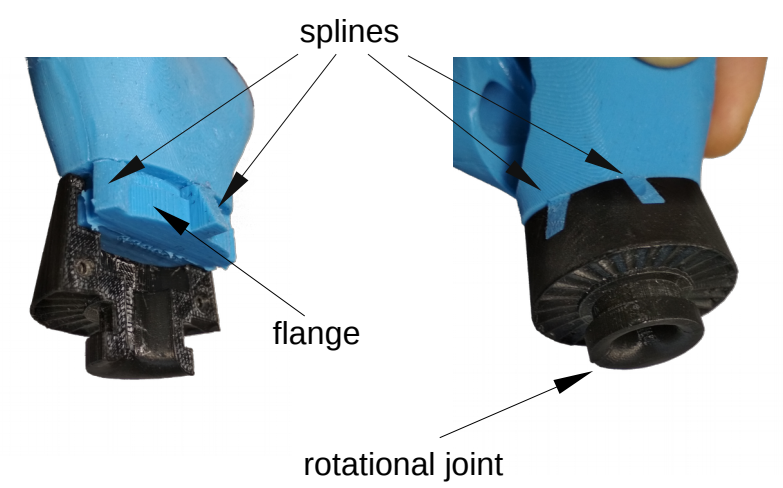

Fig. 5: Rotational hand connector.

As a prosthetic, the hand requires a proper mechanical user interface to be attached to the patient's arm. The hand itself is designed to be easily detached from such an interface (e.g. for the replacement purpose) and to enable rotation corresponding to the wrist joint. Since the hand is soft, it can not be easily attached to the interface with screws. We don't want it also to be glued since such a connection would be permanent. For that reason, a connector has been designed. The connector is based on spline and flange joints. Such an approach provides a reliable but flexible connection that can be easily detached whenever required. On the other side of the connector, there is a rotational joint that enables to change the whole hand orientation, fig. 5. The connector is hollow so that the pressure pipes can be easily guided inside. 


\section{Manufacturing}

The manufacturing process is very much similar to the process described in [8-10]. It involves several silicone moulding steps and utilizes a set of 3D printed moulds. The materials used are two silicones of different stiffness and polyester thread for the reinforcement.

\subsection{Actuators}

The process starts with preparing the thread to be embedded into the actuators structure. The thread is winded tightly onto long conical cores. When wrapped the cores are enclosed within the mould and cast with soft silicone. The mould keeps them centered and creates a thin and uniform layer of silicone that forms the external actuator wall and bounds the reinforcing thread. As the rods' diameter decrease with its length, the silicone layer with the thread inside can be easily removed from the rods towards the thinner end of the core, fig. 6 .

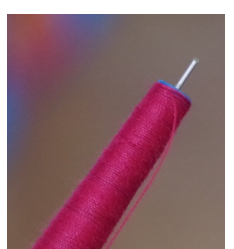

(a)

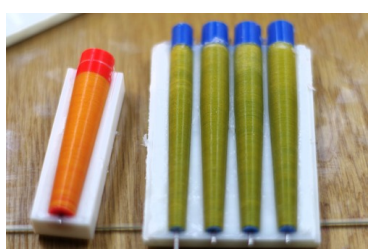

(b)

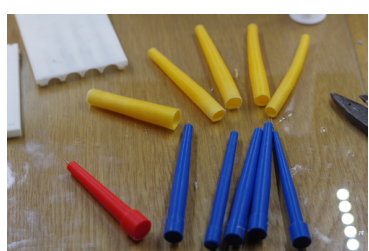

(c)

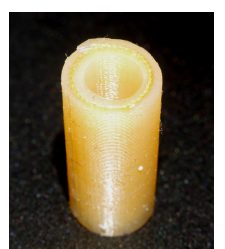

(d)

Fig. 6: Actuators manufacturing: reinforcement, external layer, cross-section reinforcement and internal layer visible.

Such conical tubes with the helical thread structure attached to the internal side are filled with a new portion of silicone and inserted into the same mould again to create another layer of silicone. The difference is that in this step smaller rods are inserted so that a certain distance inside the winding is preserved for the uncured silicone. While the silicone cures, it creates a layer on the internal side of the reinforcement. The purpose of this layer is to protect the thread from detaching the chamber surface when pressurized. Once the conical tubes are finished (fig. 6c), they are sealed on both ends to create the ready to use actuators. For that, the stiff silicone is used. The wider end of each actuator, that is its base, contains a small hole for the pressure inlet. For those holes $1.2 \mathrm{~mm}$ needle is inserted into the mould that creates the actuators enclosure. Such a diameter of the hole combined with $2 \mathrm{~mm}$ diameter flexible pipe creates an airtight and reliable connection that does not require glue. However, the tubes are glued in order to prevent them to be pulled out from the actuator, fig. 7 . 


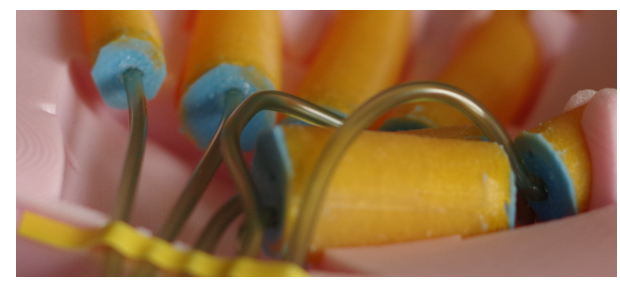

Fig. 7: Fingers bases with pressure pipes connected. The actuators' pressure inlets are $1.2 \mathrm{~mm}$ in diameter, the pipes' diameter is $2 \mathrm{~mm}$.

\subsection{Hand}

Once the set of actuators is ready, they are arranged in the mould that forms the hands' final shape. The mould ensures the joints to not be covered with the stiff silicone on the outer side that finally makes them bend into the desired direction. This is crucial to not cover the external side of the joints, otherwise, the expansion of the actuators would be constrained in the whole volume in the same way and no bending would be generated. This functionality is provided by specially designed sockets that protect the crucial areas of the actuators, fig. 8 .

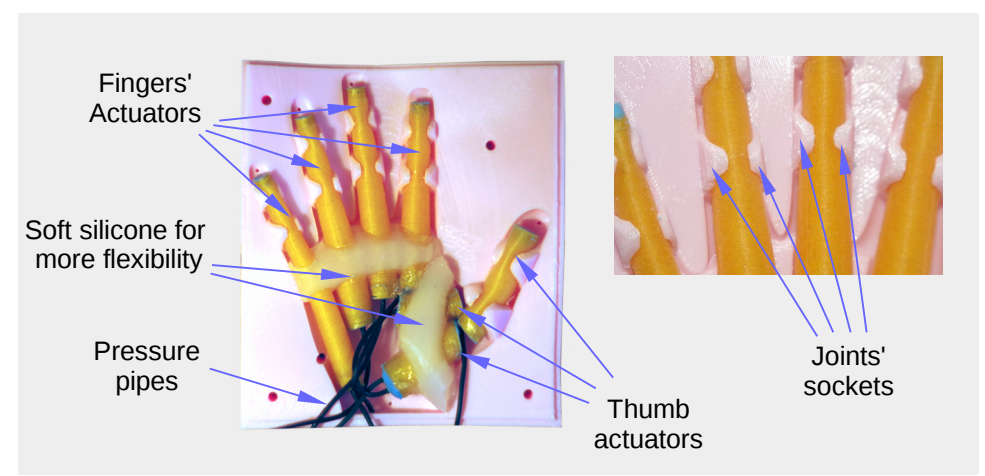

Fig. 8

The auxiliary structures made of soft silicone help to provide more flexibility where required (e.g. in between the base - metacarpophalangeal - joint of each finger and the palm surface). Before injecting of the stiff silicone all the arranged parts are glued to the mould using the same soft silicone, so they attach tightly to the mould. Thanks to that risk of penetration of the undesired areas by the stiff silicone is dramatically decreased.

When all the above is done, the mould is closed and the stiff silicone is injected into it that is the last manufacturing step. The filled mould and the hand took out from the mould is presented in fig. 9 . 

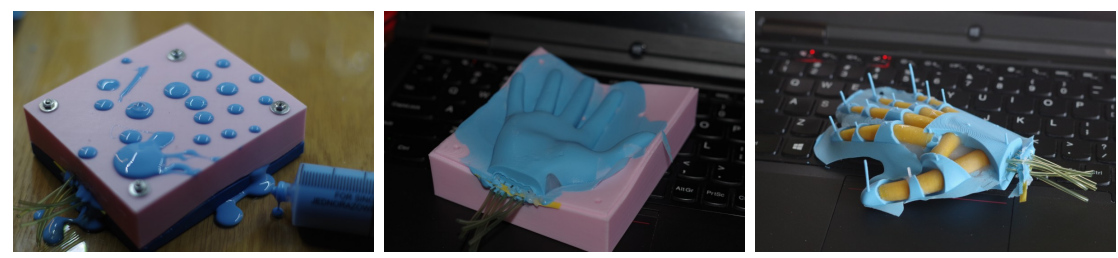

Fig. 9

After cutting off the silicone that filled the canals in the mould the hand is ready to use.

\section{Conclusions}

In this paper, we propose a soft prosthetic hand that is cheap and easy to manufacture. It is designed to be easily adjusted for individual needs of the patient and to be easily replaced in case of damage or whenever bigger size is needed. For those reasons is especially suitable for children patients as it is affordable and safe. The proposed design shows a great potential and receives very positive feedback regarding its appearance and features. Initial tests show it provides sufficient grasping capabilities to perform simple tasks. Work presented in this paper is an early prototype and has not been extensively tested yet. In the nearest future, we plan to extensively test the forces and bending angles generated by individual fingers as functions of pressure as well as the grasping and manipulation capabilities. We also consider hydraulic and pneumatic actuation. We also investigate the 3D printing as a potential technology for manufacturing of parts or whole device. Exemplary grasped objects and gestures are presented in fig. 10 .

\section{References}

1. Manuel G Catalano, Giorgio Grioli, Edoardo Farnioli, Alessandro Serio, Cristina Piazza, and Antonio Bicchi. Adaptive synergies for the design and control of the pisa/iit softhand. The International Journal of Robotics Research, 33(5):768-782, 2014.

2. Raphael Deimel and Oliver Brock. A novel type of compliant and underactuated robotic hand for dexterous grasping. The International Journal of Robotics Research, 35(1-3):161-185, 2016.

3. Huichan Zhao, Kevin O'Brien, Shuo Li, and Robert F Shepherd. Optoelectronically innervated soft prosthetic hand via stretchable optical waveguides. Sci. Robot., 1(1):eaai7529, 2016.

4. Stefan Schulz, Christian Pylatiuk, and Georg Bretthauer. A new ultralight anthropomorphic hand. In Robotics and Automation, 2001. Proceedings 2001 ICRA. IEEE International Conference on, volume 3, pages 2437-2441. IEEE, 2001. 


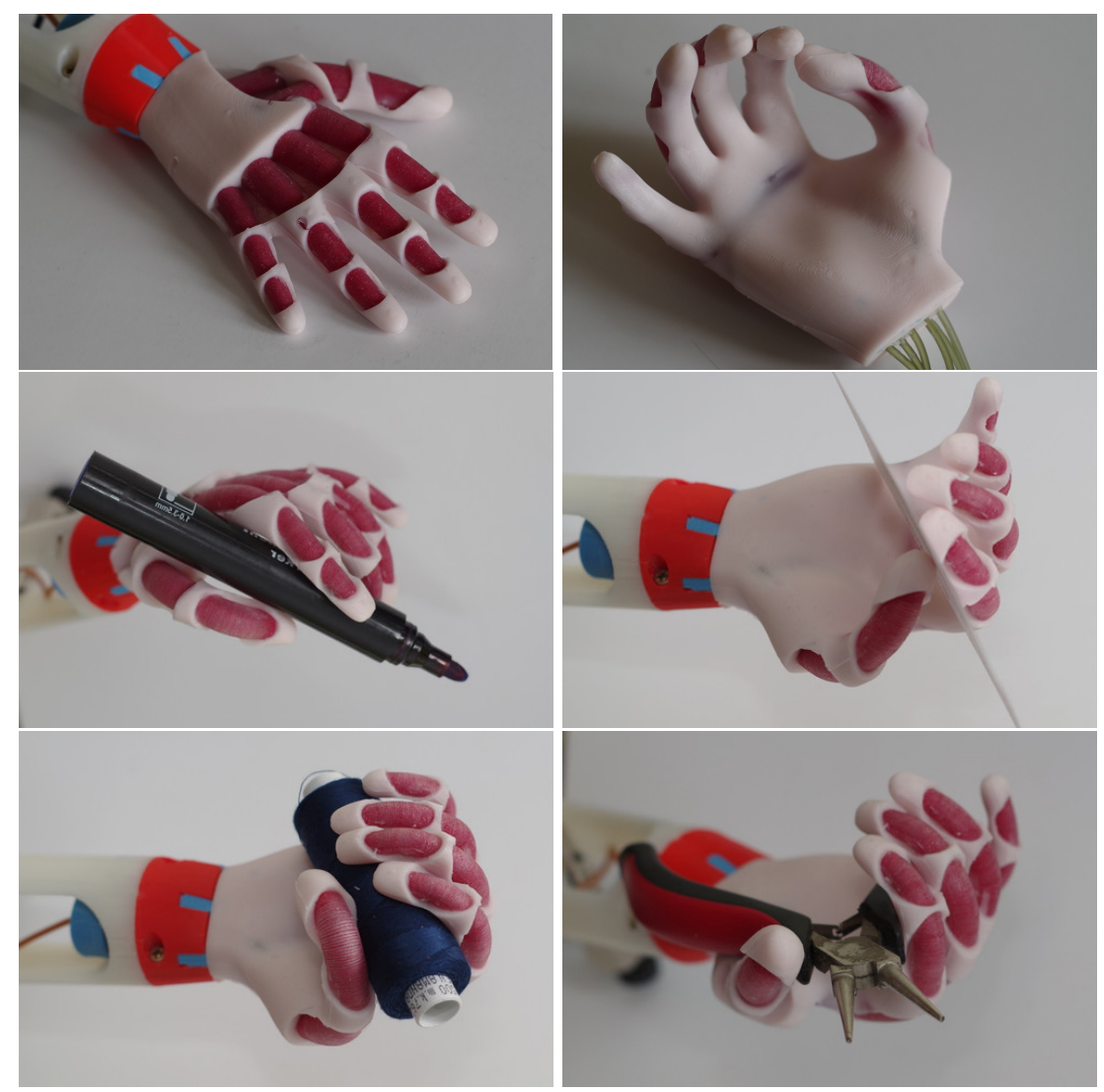

Fig. 10: Soft prosthetic hand 
5. Mahmoud Tavakoli and Aníbal T de Almeida. Adaptive under-actuated anthropomorphic hand: Isr-softhand. In Intelligent Robots and Systems (IROS 2014), 2014 IEEE/RSJ International Conference on, pages 1629-1634. IEEE, 2014.

6. Maria Chiara Carrozza, Giovanni Cappiello, Giovanni Stellin, Franco Zaccone, Fabrizio Vecchi, Silvestro Micera, and Paolo Dario. A cosmetic prosthetic hand with tendon driven under-actuated mechanism and compliant joints: ongoing research and preliminary results. In Robotics and Automation, 2005. ICRA 2005. Proceedings of the 2005 IEEE International Conference on, pages 2661-2666. IEEE, 2005.

7. Mike Kaczkowski. Cosmesis is much more than appearance... it's function. inMotion, 9:1-48, 1999

8. J. Fras, M. Macias, F. Czubaczynski, P. Salek, and J. Glowka. Soft flexible gripper design, characterization and application. In International Conference SCIT, Warsaw, Poland. Springer, 2016.

9. J. Fras, Y. Noh, H Wurdemann, and K. Althoefer. Soft fluidic rotary actuator with improved actuation properties. In International Conference on Intelligent Robots and Systems. IEEE, 2017.

10. J. Fras, J. Czarnowski, M. Macias, J. Glowka, M. Cianchetti, and A. Menciassi. New stiff-flop module construction idea for improved actuation and sensing. In International Conference on Robotics and Automation, pages 2901-2906. IEEE, 2015 . 